\title{
First Observation of Rotation Acceleration of Magnetic Island by Using Rotating Resonant Magnetic Perturbation on the J-TEXT Tokamak
}

\author{
B Rao $^{1,2}$, Y H Ding ${ }^{1,2}$, Q M Hu ${ }^{1,2}$, N C Wang ${ }^{1,2}$, B Yi ${ }^{1,2}$, M Y Zheng ${ }^{1,2}$, W Jin ${ }^{1,2}$,

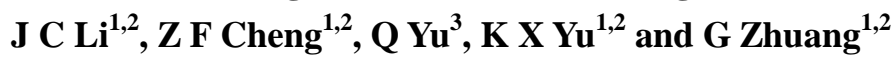 \\ ${ }^{1}$ State Key Laboratory of Advanced Electromagnetic Engineering and \\ Technology (Huazhong University of Science and Technology), Wuhan 430074, \\ China \\ ${ }^{2}$ College of Electrical and Electronic Engineering (Huazhong University of \\ Science and Technology), Wuhan 430074, China \\ ${ }^{3}$ Max-Planck-Institut für Plasmaphysik, EURATOM Association, 85748 \\ Garching, Germany \\ E-mail: yhding@mail.hust.edu.cn
}

\begin{abstract}
A set of in-vessel saddle coils called dynamic resonant magnetic perturbation (DRMP) for generating rotating resonant magnetic perturbations has recently been constructed on the J-TEXT tokamak. The phenomenon of tearing mode locking to DRMP and rotating together with the DRMP field has been observed. There is an apparent decrease of the island width during the locking and unlocking procedure. Similar results are obtained in the numerical simulation.
\end{abstract}

\section{Introduction}

Tearing mode is a very common instability in magnetically confined plasma, and how to avoid plasma disruption caused by tearing mode is a key issue in tokamak research and future fusion reactors. Tearing mode leads to the formation of magnetic island, which normally rotates in the toroidal direction. A large tearing island will greatly decrease plasma confinement. More seriously, the island may be locked to the intrinsic error field of the tokamak device and cause major plasma disruption [1]. Therefore, a proper control method should be developed to prevent the magnetic island locking to the error field or even suppress the tearing mode. Externally exerted resonant magnetic perturbation (RMP) can interact with the tearing mode directly and change its behavior. According to the experimental results in the past years, the static resonant magnetic perturbation (RMP) can suppress the tearing mode to a very small level. However, its effectiveness heavily depends on the initial state of the tearing mode, such as mode frequency and island width. In some cases, the static RMP will lead to mode locking [2-6]. Furthermore, an RMP that is too strong can stimulate a tearing mode even if the initial plasma is tearing stable [7, 8].

Compared with the static RMP, a rotating RMP is more controllable in terms of frequency and phase of the external field. According to experimental results on ASDEX-U, the tearing mode can be stabilized by a modulated electron cyclotron current drive (ECCD) [9]. If the island is locked to a known rotating RMP, the frequency and position of the island is controllable, and the ECCD stabilization can be more efficient [10]. Moreover, the rotating RMP having a changing frequency could prevent the island from locking to the RMP and suppress the island [11]. Early experiments to study effects of rotating RMP on the tearing mode were conducted on ATC [12], TO-1 [13] and DITE tokamak[14]. On HBT-EP, the frequency modulation of RMP is found to be helpful for tearing mode stabilization, in which the ion flow damping was found to play an 
important role [15]. Numerous findings on rotating RMP on TEXTOR in the fields of plasma rotation, tearing mode suppression, edge radial electric field and edge plasma transport are reported over the past several years $[16,17]$. In this paper, magnetic island locking to the rotating RMP is also observed on the J-TEXT tokamak, and corresponding numerical simulations are conducted.

\section{Rotation driving of the magnetic island by rotating RMP}

$\mathrm{J}$-TEXT is a circular cross-section tokamak with a major radius $\mathrm{R}_{0}=1.05 \mathrm{~m}$ and minor radius a $=0.265 \mathrm{~m}$. On J-TEXT, a set of dynamic resonant magnetic perturbation (DRMP) coils were newly constructed [18,19]. DRMP consists of 12 saddle coils divided into 4 groups and installed at 4 toroidal equivalent locations, as shown in Figure 1. To reduce the shielding effect of the vessel wall on the magnetic field, the coils are installed inside the vacuum vessel wall. DRMP is designed operating in a frequency range from $800 \mathrm{~Hz}$ to $10 \mathrm{kHz}$, which covers the typical tearing mode frequencies on J-TEXT. The three coils in the same group are designed at the upside, outside and the downside of the vessel. To produce a large $m / n=2 / 1$ ( $m$ and $n$ are the poloidal and toroidal mode numbers, respectively) component of the rotating magnetic perturbation, the current directions of the three coils in the same group are always set as "+- +". In addition, the phases of the current in the four groups are configured leading or lagging $90^{\circ}$ successively.

The magnetic field on the edge magnetic surface produced by DRMP is calculated by using the finite element method taking the eddy current in the wall into account. The mode structure is analyzed by applying Fourier transform to the radial part of the magnetic field. During J-TEXT discharges, the directions of plasma current and toroidal field are both counterclockwise in the top view. Therefore, it is necessary for $\mathrm{m}$ and $\mathrm{n}$ to have the same sign to be resonant with the plasma. The rotating components with the same sign of $m$ and $n$ are shown in Figure 2, in which the operation frequency is set as $5 \mathrm{kHz}$, and the four groups of coils are configured in the lagging mode. The components of $n=1,3,5,7$ and 9 are much larger compared with those of the others. The edge safety factor of J-TEXT discharge is normally set at approximately 3.5. The high $\mathrm{n}$ modes such as $\mathrm{n}=3,5,7$ and 9 components cannot be resonant with plasma. Compared with resonant components, the non-resonant components have much smaller effects on the tearing mode, and can be usually neglected in the simplified analysis. Consequently, the component which rotates in the direction of $-(2 \theta+\varphi)$, which is expressed as $b_{\mathrm{r} 21} \cos (2 \theta+\varphi+\omega t)$, is the main resonant component under the aforementioned coil configuration. The amplitude of the resonant $2 / 1$ component of the field on the edge magnetic surface is approximately 0.45 Gauss per $1 \mathrm{kA}$ current.

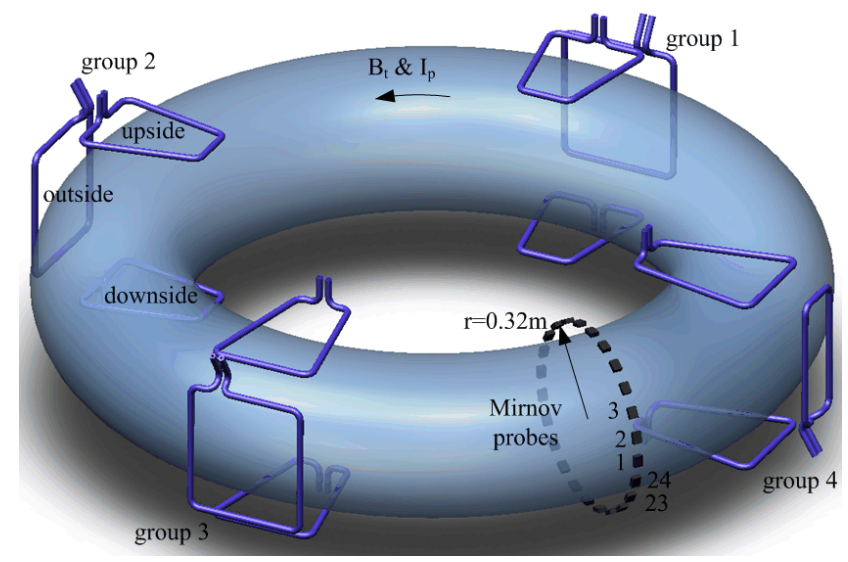


Figure 1. Layout of the DRMP coils and poloidal array of Mirnov probes on J-TEXT. The three coils located at the same toroidal location form a group. The four groups are successively placed differing $90^{\circ}$ in the toroidal direction.
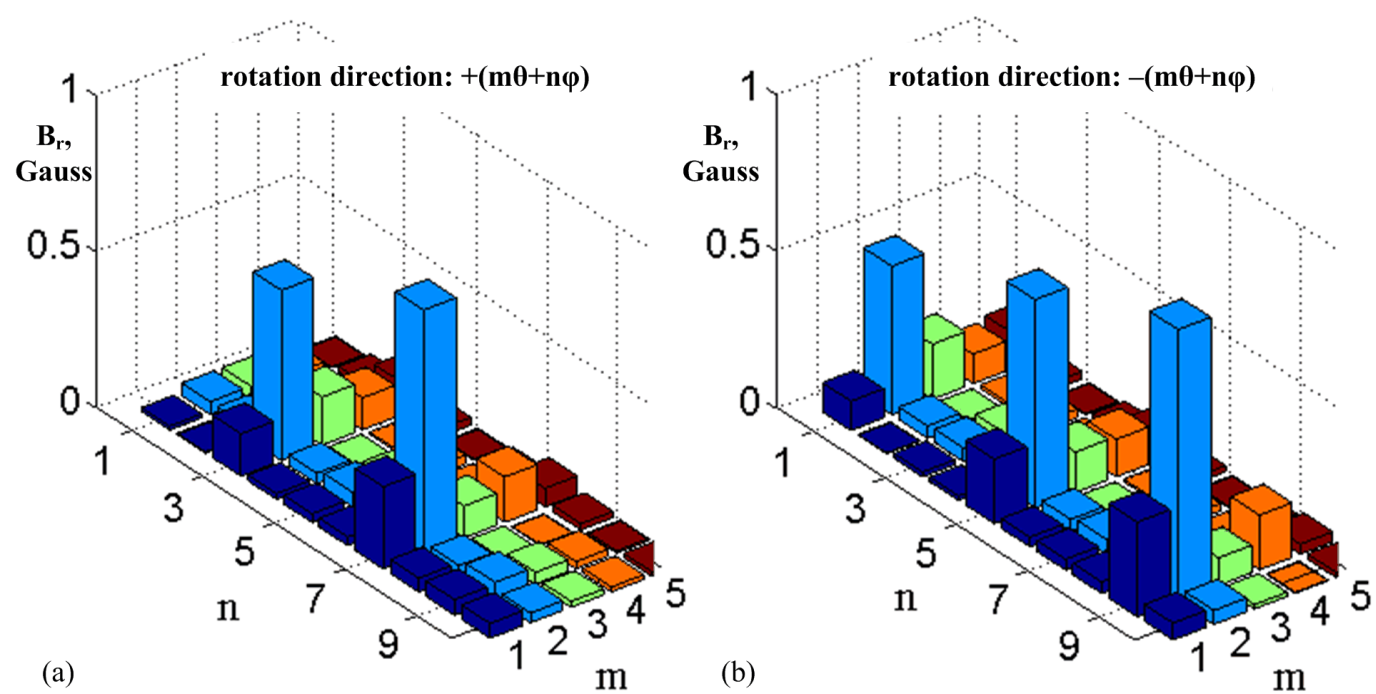

Figure 2. Mode structure of the radial field caused by DRMP coils. Only components with the same sign of $\mathrm{m}$ and $\mathrm{n}$, which are possible resonant with the J-TEXT plasma under the typical configuration of the equilibrium field, are given in the figure. The magnetic field is calculated without taking the plasma response into account.

An existing tearing mode is very likely to be locked to the rotating RMP and then rotates synchronously with the external field. A typical process of mode locking to the DRMP field on J-TEXT is shown in Figure 3, in which the plasma current, currents of DRMP coils, $\mathrm{dB}_{\theta} / \mathrm{dt}$, edge plasma rotation, frequency evolution and island width are given. The plasma current and the toroidal field are set as $200 \mathrm{kA}$ and $1.8 \mathrm{~T}$, respectively. The coils of groups 1 and 3 are connected in series and fed with an AC power supply whose current is $\mathrm{I}_{\text {DRMP1}}$, while groups 2 and 4 are fed with another power supply with current $\mathrm{I}_{\mathrm{DRMP} 2}$. The phase of $\mathrm{I}_{\mathrm{DRMP} 1}$ lags $\mathrm{I}_{\mathrm{DRMP} 2} 90$ degrees, and then the RMP is rotating in the counter plasma current direction. The DRMP operates at $5 \mathrm{kHz}$ and the frequency remains unchanged throughout the discharge. After the DRMP is switched on at $0.25 \mathrm{~s}$, the mode frequency, namely $\mathrm{f}_{\text {mirnov }}$, which is calculated by performing time-frequency analysis on $\mathrm{dB}_{\theta} / \mathrm{dt}$, starts to increase as the island width decreases. The island width is considered proportional to the square root of $A_{\text {mirnov }} / f_{\text {mirnov }}$, where $A_{\text {mirnov }}$ is the amplitude obtained from the time frequency analysis of $\mathrm{dB}_{\theta} / \mathrm{dt}$. When the current of DRMP reaches a threshold, approximately $0.6 \mathrm{kA}$ in this shot, the mode frequency jumps abruptly from $4.5 \mathrm{kHz}$ to $5 \mathrm{kHz}$, which is the same with the operation frequency of the power supply as well as the rotation frequency of the externally exerted RMP. However, the island width recovers quickly to the initial level after being locked. Then the mode frequency remains at $5 \mathrm{kHz}$ until the DRMP current drops to a small level, at which point the island is unlocked from the rotating RMP. Meanwhile, the edge plasma rotation measured by the Doppler shift of the spectrum of $\mathrm{C}-\mathrm{V}$ radiation, changes toward the counter-current direction. The edge rotation recovers again after the island is unlocked. Other parameters such as electron density, electron temperature and soft X-ray radiation, change little during the locked phase. When the current of the DRMP coil decreases, the island unlocks. There is an apparent decrease of the island width during the locking and unlocking procedure, which was also observed in [15]. It should be noted that the magnetic field of DRMP contributes little to the Mirnov signals because the Mirnov array locates relatively far from the DRMP coils. 


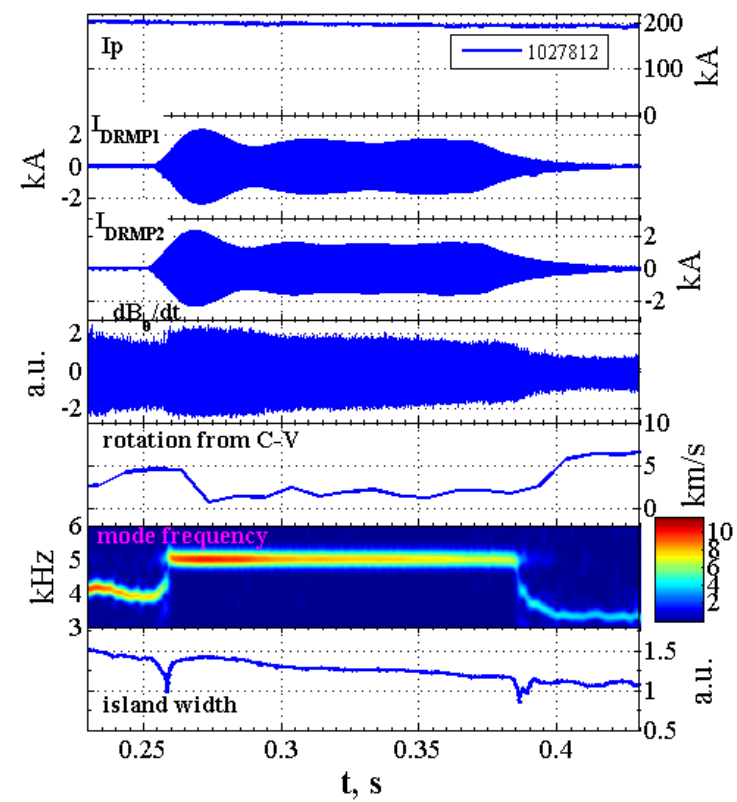

Figure 3. Signals including plasma current, current of DRMP, $\mathrm{dB}_{\theta} / \mathrm{dt}$, edge plasma rotation, mode frequency and island width in a typical mode locking process to DRMP. The magnetic island is locked to DRMP at $0.26 \mathrm{~s}$ and unlocked at $0.385 \mathrm{~s}$. The color in the frequency panel stands for the strength of a certain frequency component of the Mirnov signal.

The evolution of the phase difference between the island and the RMP during the locking procedure is shown in Figure 4. The 2/1 poloidal magnetic field caused by the RMP or the island

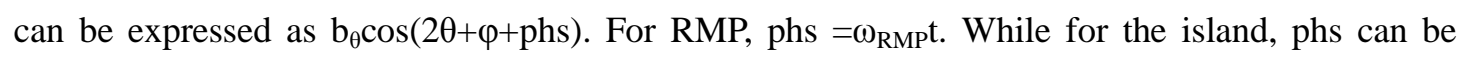
obtained by finding the summit of the integration signal of Mirnov signals at a certain time. From the figure it can be seen that, before locked, the phase difference varies from 0 to $2 \pi$, while at about $0.259 \mathrm{~s}$, the island is suddenly locked and then rotates together with the RMP.
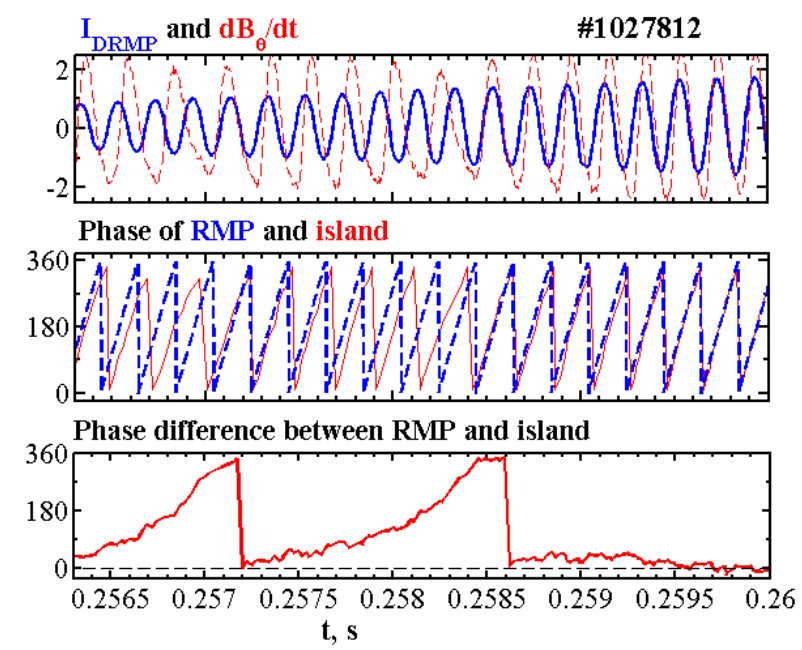

Figure 4. The evolution of the phases of the RMP, the island and their difference.

A comparison between two shots with different frequencies and initial islands width is given in Figure 5. In shot 1027812, the operation frequency of DRMP is set as $5 \mathrm{kHz}$, while it is $6 \mathrm{kHz}$ in shot 1027817 . The initial mode frequency in shot 1027812 is approximately $4 \mathrm{kHz}$. The island locks to the DRMP field after the DRMP is turned on, whereas in shot 1027817, the island with an initial mode frequency of about $2.5 \mathrm{kHz}$ is not locked. Although the island was not locked to the 
DRMP field, its frequency has an increase and the island width decreases gradually under the influence of DRMP. Besides, in shot 1027817, the signal of island width after 0.35s becomes meaningless, because the calculation method no longer works in the period when the Mirnov signal has a large distortion. Whether or not the island can be locked to the DRMP field seems to be related to the island width and the frequency difference between the island and the rotation field. In [10], the island locking threshold to an external static RMP has been studied in detail, and is given as $\psi_{\text {th }} \propto \mu \omega\left(V_{A} \mathrm{~W} / \mathrm{a}\right)^{-2}$, where $\mu$ is the plasma viscosity and $V_{A}$ is the Alfven velocity. When the RMP is rotating, $\omega$ stands for the frequency difference between the island and the RMP. In these two shots, the frequency diference are $3.5 \mathrm{kHz}$ and $1 \mathrm{kHz}$, and the relative initial island widths are 1.8 and 1.25, respectively. According to [10], the ratio of required perturbation fields will be about $3.5 / 1 \times 1.25^{2} / 1.8^{2}=1.7$, which indicates that it is more difficult for the island in shot 1027817 to be locked.

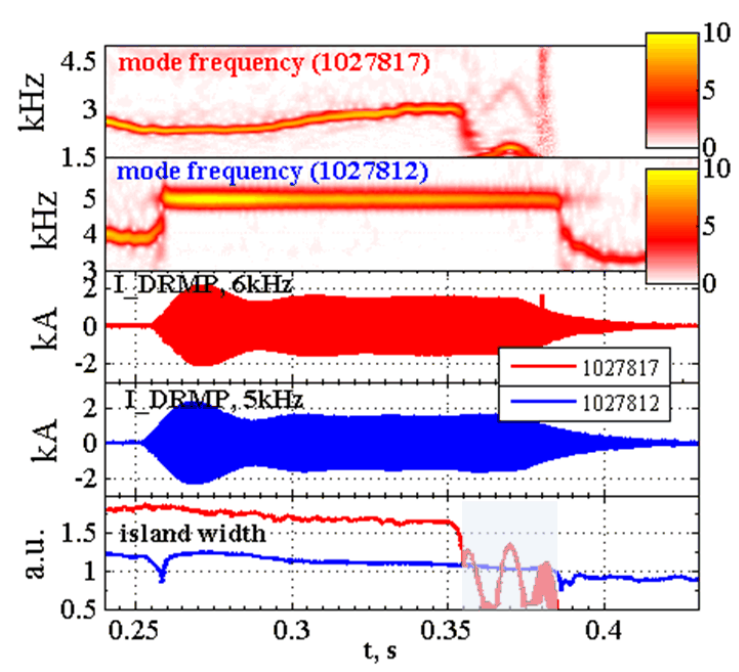

Figure 5. A comparison of the mode frequency and the island width between two shots with and without mode locking. The frequencies of the rotating magnetic perturbation are $6 \mathrm{kHz}$ and $5 \mathrm{kHz}$ respectively for shots 1027817 and 1027812, respectively. The color in the frequency panel stands for the strength of a certain frequency component of the Mirnov signal.

\section{Numerical modeling}

To understand the different experimental results shown in Figures 3 and 5, a nonlinear numerical model based on reduced MHD equations was used. In the model low $\beta$ and large aspect-ratio approximations are utilized. The magnetic field is defined as $\boldsymbol{B}=\mathrm{B}_{\mathrm{t}} \boldsymbol{e}_{\mathrm{t}}-\mathrm{B}_{\theta} \boldsymbol{e}_{\theta}-\nabla \psi \times \boldsymbol{e}_{\mathrm{t}}$, where $\psi$ is the helical flux function. The basic equations utilized are Ohm's law and the equation of motion. Normalizing the length to minor radius a, the time $t$ to the resistive time $\tau_{R}=a^{2} \mu_{0} / \eta$, the helical flux $\psi$ to $a B_{t}$, plasma velocity $v$ to $a / \tau_{R}$, and the toroidal plasma current density $j$ to $B_{t} / a$, these equations become

$$
\begin{aligned}
& \frac{\mathrm{d} \psi}{\mathrm{d} t}=E_{0}-\eta j, \\
& \frac{\mathrm{d} U}{\mathrm{~d} t}=-S^{2} \nabla_{\|} j+\mu_{\perp} \nabla_{\perp}^{2} U+S_{m},
\end{aligned}
$$

where $\mathrm{d} / \mathrm{dt}=\partial / \partial \mathrm{t}+\mathrm{v}_{\perp} \cdot \nabla, \mathrm{j}=-\nabla_{\perp}^{2} \psi-2 \mathrm{nB}_{\mathrm{t}} /(\mathrm{mR})$ is the toroidal plasma current density. $\eta$ is the normalized plasma resistivity, and $\mathrm{E}$ is the equilibrium electric field for maintaining the equilibrium plasma current. The magnetic Reynolds number $S=\tau_{R} / \tau_{A}$, where $\tau_{A}=a / V_{A}$ is the 
toroidal Alfvén time. $U=-\nabla_{\perp}^{2} \phi$ is the plasma vorticity, and $\mu$ is the plasma viscosity. $S_{m}$ in equation (2) is the poloidal momentum source which leads to an equilibrium plasma rotation.

The effect of a single helicity RMP with $\mathrm{m} / \mathrm{n}=2 / 1$ is taken into account by the boundary condition $\psi_{2 / 1}(\mathrm{r}=\mathrm{a})=\psi_{\mathrm{a}} \mathrm{aB} \mathrm{t} \cos \left(\mathrm{m} \theta+\mathrm{n} \varphi+2 \pi \mathrm{f}_{\text {mode }} \mathrm{t}\right)$, where $\psi_{\mathrm{a}}$ describes the normalized $\mathrm{m} / \mathrm{n}=2 / 1$ helical magnetic flux amplitude at $\mathrm{r}=\mathrm{a}, \theta$ and $\varphi$ are the poloidal and toroidal angle, respectively. The radial magnetic field perturbation at $r=a$ is given by $b_{r a}=m \psi_{a} B_{t}$. Eqs. (1) and (2) are solved simultaneously using the initial value code TM1, which has been used for modeling the nonlinear growth and saturation of NTMs [21], their stabilization by RF current [10] and the effect of RMP on resistive TM [5].

To compare with experimental results shown in section 2, the input parameters for following calculations are based on J-TEXT experimental parameters. A monotonic profile for safety factor $q$ is taken with $q=2 / 1$ surface located at $0.7 \mathrm{a}$. The electron temperature is about $300 \mathrm{eV}$ at the $q=$ 2 surface, and the local electron density is about $1 \times 10^{19} \mathrm{~m}^{-3}$. These parameters lead to magnetic Reynold number $S=6 \times 10^{6}$ and resistive time $\tau_{R}=0.2 \mathrm{~s}$. Plasma viscosity is assumed to be at the anomalous transport level $\mu_{\perp}=0.5 \mathrm{~m}^{2} \mathrm{~s}^{-1}$.

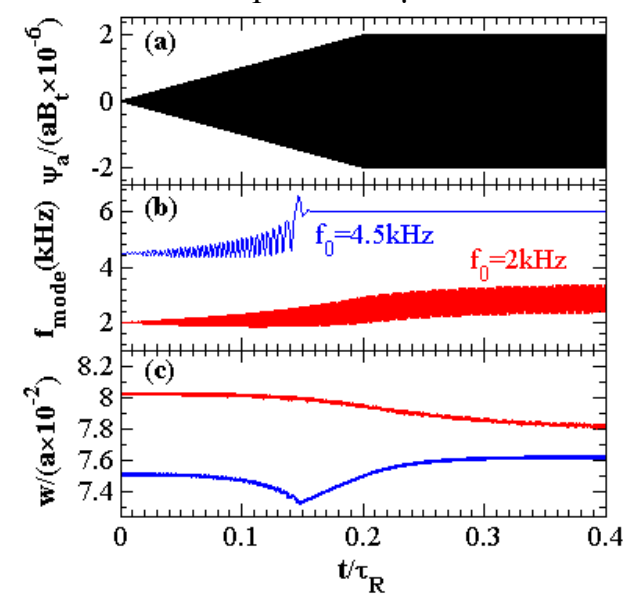

Figure 6. Time evolution of normalized (a) RMP amplitude $\psi_{\mathrm{a}}$, (b) mode frequency $f_{\text {mode }}$ and (c) island width w/a. The rotating frequency of 2/1 RMP is $6 \mathrm{kHz}$, the two initial mode frequencies are $2 \mathrm{kHz}$ (red curve) and $4.5 \mathrm{kHz}$ (blue curve), respectively.

In figure 6 of the numerical results, the time evolutions of the normalized (a) 2/1 RMP amplitude $\psi_{\mathrm{a}}$, (b) mode frequency $f_{\text {mode }}$ and (c) island width w/a are shown. The initial mode frequencies are set as $f_{0}=2 \mathrm{kHz}$ and $4.5 \mathrm{kHz}$, respectively, and the corresponding saturated island width are $0.08 \mathrm{a}$ and $0.075 \mathrm{a}$ as shown in figure 6(c). The frequency of the applied rotating RMP is set as $6 \mathrm{kHz}$, the RMP amplitude linearly increases with time during $0 \leq \mathrm{t} \leq 0.2 \tau_{\mathrm{R}}, \psi_{\mathrm{a}}=2 \times 10^{-6} \mathrm{aB}_{\mathrm{t}}$ at $t=0.2 \tau_{R}$, for $t>0.2 \tau_{R}$, the amplitude keeps constant as shown in figure $6(\mathrm{a})$, similar to experimental setup. For lower mode frequency case $\left(f_{0}=2 \mathrm{kHz}\right)$, the mode frequency is driven from $2 \mathrm{kHz}$ to $3 \mathrm{kHz}$, and the island width decreases slightly as shown in figure 6(b). While for higher mode frequency case $\left(f_{0}=4.5 \mathrm{kHz}\right)$, when the RMP amplitude reaches $1.5 \times 10^{-6} \mathrm{aB}_{\mathrm{t}}$ at $\mathrm{t}=$ $0.15 \tau_{\mathrm{R}}$, mode locking happens and the mode frequency experiences a large jump and then keeps constant of $6 \mathrm{kHz}$. Before mode locking, the island width decreases obviously, while after mode locking, the island width grows and saturates with $\mathrm{w}=0.076 \mathrm{a}$, a little larger compared with the initial width. The results shown in figure 6 approximately agree with experimental results as shown in section 2. Further calculations indicate that the required $\psi_{\mathrm{a}}$ for mode locking is proportional to the plasma viscosity $\mu$ for large $\mu$, but its dependence on $\mu$ is weak for small $\mu$, 
consistent with the results in [10].

\section{Summary and discussion}

Magnetic island locking to the external rotating RMP was observed on the J-TEXT tokamak. The island can be driven by the RMP to a higher rotation velocity. The island width is found having an obvious decrease during both the locking and unlocking phase. After locking, the island width recovers to the initial value or reaches a larger level. The numerical results approximately agree with experimental ones. These results together with previous experimental findings [4-6] suggest that a rotating RMP could be utilized to increase the island rotation frequency for avoiding mode locking to the intrinsic error field of the tokamak. As the external RMP has a strong suppression effect on the island width during the locking procedure, it provides a possible control method of tearing mode if the RMP frequency can be kept to be larger than that of the magnetic island. More detailed research on the interactions between the tearing mode and external RMP, such as behaviors of the island under different plasma parameters and possible feedback control of the tearing mode, will be undertaken in the succeeding works.

\section{Acknowledgements}

This work is supported by the National ITER Project Foundation of China (under grant nos. 2010GB107004 and 2011GB109001) and the National Natural Science Foundation of China (No. 11275080)

\section{References}

[1] Nave M F F and Wesson J A 1990 Nucl. Fusion 302575

[2] Hender T C, et al 1992 Nucl. Fusion 322091

[3] Elgriw S, et al 2011 Nucl. Fusion 51113008

[4] Rao B, et al Physics Letters A 377 315-318

[5] Hu Q M, et al 2012 Nucl. Fusion 52083011

[6] Jin W, et al 2013 Plasma Phys. Control. Fusion 55035010

[7] Buttery R J, et al 1999 Nucl. Fusion 391827

[8] Wolfe S M, et al 2005 Phys. Plasmas 12056110

[9] Maraschek M, et al 2007 Phys. Rev. Lett. 98025005

[10] Yu Q and Günter S 2008 Nucl. Fusion 48065004

[11] Lazzaro E and Nave M F F 1988 Phys. Fluids 31 1623-1629

[12] Bol K et al 1975 Proc. 5th Int. Conf. on Plasma Physics and Controlled Nuclear Fusion Research 1975 (Tokyo, 1974) vol 1 (Vienna: IAEA) p 83

[13] Ivanov N V and Kakurin A M 1979 Sov. J. Plasma Phys.5 541

[14] Morris A W, et al 1990 Phys. Rev. Lett. 64 1254-1257

[15] Navratil G A, et al 1998 Phys. Plasmas 51855

[16] Wolf R C, et al 2005 Nucl. Fusion 451700

[17] Coenen J W, et al 2011 Nucl. Fusion 51063030

[18] Rao B, et al Introduction to Resonant Magnetic Perturbation Coils of the J-TEXT Tokamak. (submitted to Fusion Engineering and Design)

[19] Rao B, et al 2012 IEEE Trans. on Applied Superconductivity 224201804

[20] Fitzpatrick R 1993 Nucl. Fusion 331049

[21] Yu Q, Günter S, and Lackner K 2004 Phys. Plasmas 11140 